\title{
Factors Impacting Pathologic Complete Response after Neoadjuvant Chemotherapy in Breast Cancer: A Single-Center Study
}

\author{
Asiie Olfatbakhsh, ${ }^{1,}{ }^{*}$ Hoda Tafazzoli-Harandi, ${ }^{1}$ Safa Najafi, ${ }^{1}$ Esmat Al Sadat Hashemi, ${ }^{1}$ Fateme Sari, ${ }^{1}$ \\ Parisa Mokhtari Hesari, ${ }^{2}$ and Parisa Hosseinpour ${ }^{1}$ \\ ${ }^{1}$ Breast Disease Department, Breast Cancer Research Center, Motamed Cancer Institute, ACECR, Tehran, IR Iran \\ ${ }^{2}$ Integrative Medicine Department, Breast Cancer Research Center, Motamed Cancer Institute, ACECR, Tehran, IR Iran \\ "Corresponding author: Asiie Olfatbakhsh, Breast Disease Department, Breast Cancer Research Center, Motamed Cancer Institute, ACECR, Tehran, IR Iran. Tel: +98-2188797543; \\ +98-9121590381, Fax: +98-2188796208, E-mail: folfatbakhsh@yahoo.com
}

Received 2017 November 28; Revised 2018 February 04; Accepted 2018 February 19.

\begin{abstract}
Background: Neoadjuvant chemotherapy is the standard treatment for patients with locally advanced breast cancer which was recently introduced for operable breast cancer especially to achieve negative margins in breast conservation. Several studies have shown that Pathologic complete response (pCR) after neoadjuvant chemotherapy increases survival rate. The aim of this study therefore, was to evaluate the rate of pathologic complete response and its effective factors in breast cancer research center (BCRC). Methods: During a cross-sectional study, 179 patients with stage I to III breast cancer, who received neoadjuvant chemotherapy in breast cancer research center from 1997 to 2014, were included. Cases with pathologic complete response were defined as no tumor residue in the breast tissue and axillary region. This group of patients was compared with patients who had residual tumor at pathology. Data were analyzed by descriptive and inferential statistics using SPSS 19.

Results: The mean age of patients was 45.4 years. Thirty-four patients (19\%) were identified in the pathological complete response group (pCR). There was no significant difference between the PCR and non-pCR groups with respect to Age, Menopausal status, Family history of breast cancer, Tumor size, Histological type, Hormone receptors, Her-2neu and Phenotypic subtypes. However, ki67 index was significantly different between the two groups of patients, indicating that in patients with Ki67 of more than 40 , pCR was the most observed $(\mathrm{P}=0.01)$.

Conclusions: This study showed that among the demographic, clinical, pathological and therapeutic factors, Ki67 can be a predicting factor for pathologic complete response after neoadjuvant chemotherapy.
\end{abstract}

Keywords: Breast Neoplasms, Neoadjuvant Therapy, Ki67

\section{Background}

Neoadjuvant chemotherapy (NAC) or primary chemotherapy was proposed for the first time in 1970 for the treatment of patients with locally advanced breast cancer who had in-operable tumors. Subsequent studies including national surgical adjuvant breast and bowel project (NSABP) 18 showed that chemotherapy before surgery increases the chance of breast conservation and as such, can be introduced for tumor size reduction in operable patients.

Although the primary goal of treatment was operability, but further studies demonstrated that overall and disease free survivals were equal in patients who had surgery before chemotherapy. The most important findings of these studies are NSABP B-18T, European organization for research and treatment of cancer (EORTC) 10902 and Eu- ropean cooperative trial in operable breast cancer (ECTO) in which NAC and adjuvant chemotherapy were compared. Thus, it was shown that the overall survival was similar and the chance of breast conservation was high; however, morbidity was low in NAC group. These findings were published in a Meta-analysis in 2005 (1).

One of the potential advantages of NAC chemotherapy is that it treats the systemic micro-metastatic disease from the beginning. Also, it helps in the investigation of the effect of chemotherapy drugs and new treatment strategies in clinical trials. The neoadjuvant trial model provides rapid assessment of short-term drug efficacy utilizing pathologic complete response as the primary endpoint (2).

Although approximately $70 \%$ of patients show response in clinical examination and imaging, but only $3 \%$ $30 \%$ show pathologic complete response (pCR) according 
to previous studies. The rate of this response helps in determining the patients' prognosis and as such, pCR can improve disease free (3-6).

In a meta-analysis, the results of 12 clinical trials in patients who were treated with NAC showed that pCR after NAC is an important factor to improve disease free survival and this survival benefit was obtained in all subtypes of breast cancer. However, this relationship was stronger in patients with high-grade tumors than patients with low or intermediate grade with positive hormone receptor (7).

According to the RECIST (response evaluation criteria in solid tumors) used for evaluating the response of solid tumors a reduction of at least $30 \%$ in tumor size is considered as the treatment response. These tumors could be evaluated using imaging techniques such as computed tomography(CT) scan, magnetic resonance imaging (MRI) and ultrasound (US) before and after treatment (8). But pathologic response is the most preferred method in breast cancer. Different studies have distinct definitions for pCR, but typically, pCR is defined as: 1) the absence of invasive tumor in the breast tissue and 2) cancer cells not found in the lymph nodes. In situ carcinoma is not considered as residue because various studies have shown that it had no effect on survival (3). However, the predictors of complete response to NAC are not absolutely clear. According to previous studies, tumors with high proliferation rate and without hormone receptors expression are more sensitive to chemotherapy and are more likely to be in pCR group. In contrast, well differentiated tumors with low proliferation rate and expression of hormone receptors are less likely to show pCR (2). Ring et al. (9) reported that patients with negative estrogen receptor were more likely to achieve PCR than patients with positive estrogen receptor. In a study by Andrade et al. (10) in which Adriamycin-Cyclophosphamide chemotherapy regimen and subsequently paclitaxel were used, pCR was observed in $17 \%$ of patients especially in triple negative cases. Similar results have been reported in studies by Arvil et al. and Ring et al. $(8,9)$.

Several studies have been conducted to identify the predictor factors of PCR after NAC, however, the value of them is still uncertain, as there are some conflicting results in the literature. Therefore, the aim of this study was to assess the rate of pCR after NAC and factors affecting its rate in breast cancer patients who were referred to the breast cancer research center (BCRC) in Tehran, Iran.

\section{Methods}

This retrospective study was conducted on patients with breast cancer who referred to breast cancer research center(BCRC), Motamed cancer institute affiliated to Jahad
Daneshgahi (ACECR), Tehran, Iran, during a sixteen-year period (2007 - 2013). Inclusion criteria were stage I to III patients who had received NAC and underwent breast and axillary surgery at BCRC. Subjects with distant metastasis and also cases with inaccessible pathology results were excluded. Patients with absence of invasive tumor residue in the breast and axillary tissue were in group pCR and those with invasive residue in their tissue were divided in group non-pCR. The two groups were compared in terms of age, menopausal status, family history of breast cancer, tumor size before NAC (based on ultrasound or physical examination), tumor histology, grade, hormone receptor status including Estrogen receptor (ER) and Progestrone receptor (PR), Her2neu (Human epidermal growth factor receptor), Ki67, phenotypic subtype, type of chemotherapy regimen and the number of NAC courses. This study was accepted in scientific committee of BCRC and received the ethics committee approval (code No.IR.ACECR.IBCRC.REC.1394.59).

\subsection{Statistical Analysis}

Based on the similar projects' results (11), by estimating $\mathrm{pCR}$ rate of $17 \%(\mathrm{p}), \mathrm{q}=0.83, \mathrm{D}=0.04$, and power of $95 \%$, the number of required patients was approximated 338 persons who received neoadjuvant chemotherapy.

P value $<0.05$ was considered significant. Data analysis was performed using descriptive statistics (means and standard deviation) and categorical data were compared by t-test, chi square and fisher exact test, using SPSS 20 software.

\section{Results}

The information about 2,517 breast cancer patients since 1997 to 2013 were recorded in the database of BCRC, of them, 363 subjects had received NAC. Fifty-four patients with distant metastasis and 130 cases with incomplete data (pathology report and Immunohistochemistry results) were excluded. Overall, the data of 179 cases were analyzed. Thirty-four patients (19\%) were enrolled in the pCR group and 145 patients (81\%) in the non-pCR group. The median follow-up time was 22.1 months. The mean age of patients was $45.4 \pm 10.4$ years (25-75) and the mean of body mass index (BMI) was $28.16 \pm 4.48$. Five percent of patients had a positive family history of breast cancer in first and second degree relatives.

The clinical and pathological characteristics of patients are shown in Table 1.

The comparison of the clinic pathological characteristics between the two groups, pCR and non-pCR, is shown in Table 2.

The mean of Ki67 was 50.27 in PCR group and 31.09 in non-pCR group. There was a significant difference in terms 


\begin{tabular}{|c|c|}
\hline Variable & No. (\%) \\
\hline \multicolumn{2}{|l|}{ Tumor size } \\
\hline $\mathrm{T} 1$ & $8(5.2)$ \\
\hline $\mathrm{T} 2$ & $54(35.3)$ \\
\hline $\mathrm{T} 3$ & $62(40.5)$ \\
\hline $\mathrm{T} 4$ & $29(19)$ \\
\hline \multicolumn{2}{|l|}{ Grade } \\
\hline I & $12(8.1)$ \\
\hline II & $96(64.9)$ \\
\hline III & $40(27)$ \\
\hline \multicolumn{2}{|l|}{ Stage } \\
\hline II & $34(23.4)$ \\
\hline III & $111(76.6)$ \\
\hline \multicolumn{2}{|l|}{ Tumor histology } \\
\hline Invasive ductal carcinoma & $159(94.7)$ \\
\hline Invasive lobular carcinoma & $9(5.3)$ \\
\hline \multicolumn{2}{|l|}{ Type of surgery } \\
\hline Mastectomy & $106(64.2)$ \\
\hline Breast conserving & $59(35.8)$ \\
\hline \multicolumn{2}{|l|}{ Estrogen receptor } \\
\hline Negative & $55(34.2)$ \\
\hline Positive & $106(65.8)$ \\
\hline \multicolumn{2}{|l|}{ Progestrone receptor } \\
\hline Negative & $73(45.3)$ \\
\hline Positive & $88(54.7)$ \\
\hline \multicolumn{2}{|l|}{ Her2 new } \\
\hline Negative & $86(58.1)$ \\
\hline Positive & $62(41.9)$ \\
\hline \multicolumn{2}{|l|}{ Subtypes } \\
\hline luminal & $99(55.3)$ \\
\hline HER2+ & $24(13.4)$ \\
\hline TNBC* & $25(14)$ \\
\hline Unknown & $31(17.3)$ \\
\hline
\end{tabular}

of Ki67 level $(\mathrm{P}=0.01)$ in that, $\mathrm{pCR}$ was more observed in patients with Ki67 of more than 40 as compared with Ki67 of less than 40. Also, there was a significant difference in terms of tumor grade between the two groups $(\mathrm{P}=0.02)$. In the other words, $\mathrm{pCR}$ was less observed in patients with higher grades. The stage of disease and the number of involved lymph nodes were not considered in the study variables, because these variables were evaluated after NAC.

Furthermore, ER, PR, HER2 data were available for 148

\begin{tabular}{|c|c|c|c|}
\hline Variable & pCR Group & Non Pcr Group & P Value \\
\hline Age, $y$ & $42.27 \pm 10.19$ & $46.11 \pm 10.39$ & 0.056 \\
\hline BMI, $\mathbf{k g} / \mathrm{m}^{2}$ & $27.81 \pm 4.24$ & $28.23 \pm 4.54$ & 0.64 \\
\hline Family history & & & 0.53 \\
\hline Negative & $33(97.1)$ & $137(94.5)$ & \\
\hline Positive & $1(2.9)$ & $8(5.5)$ & \\
\hline Menopausal status & & & 0.082 \\
\hline Pre menopause & $28(82.4)$ & $96(70.1)$ & \\
\hline Post menopause & $6(17.6)$ & $47(32.9)$ & \\
\hline Tumor size, cm & & & 0.95 \\
\hline$\leq 5$ & $10(40)$ & $52(40.6)$ & \\
\hline$>5$ & $15(60)$ & $76(59.4)$ & \\
\hline Tumor histology & & & 0.91 \\
\hline $\begin{array}{l}\text { Invasive ductal } \\
\text { carcinoma }\end{array}$ & $30(93.7)$ & $130(94.9)$ & \\
\hline $\begin{array}{l}\text { Invasive lobular } \\
\text { carcinoma }\end{array}$ & $2(6.2)$ & $7(5.1)$ & \\
\hline Tumor grade & & & 0.026 \\
\hline I & $1(4.8)$ & $11(8.7)$ & \\
\hline II & $19(90.50)$ & $77(60.9)$ & \\
\hline III & $1(4.8)$ & $39(30.7)$ & \\
\hline Estrogen receptor & & & 0.45 \\
\hline Negative & $12(40)$ & $43(32.8)$ & \\
\hline Positive & $18(60)$ & $88(67.2)$ & \\
\hline Progesterone receptor & & & 0.872 \\
\hline Negative & $14(46.7)$ & $59(45)$ & \\
\hline Positive & $16(53.3)$ & $72(55)$ & \\
\hline HER 2neu & & & 0.756 \\
\hline Negative & $17(60.7)$ & $69(57.5)$ & \\
\hline Positive & $11(39.3)$ & $51(42.5)$ & \\
\hline Chemotherapy regimen & & & 0.94 \\
\hline Taxane-based & $24(72.7)$ & $99(73.3)$ & \\
\hline Non taxane-based & $9(27.3)$ & $36(26.7)$ & \\
\hline $\begin{array}{l}\text { Mean of Chemotherapy } \\
\text { courses }\end{array}$ & 6.50 & 5.93 & 0.23 \\
\hline Ki67 & & & 0.013 \\
\hline$\leq 40$ & $4(36.4)$ & $34(75.6)$ & \\
\hline$>40$ & $7(63.6)$ & $11(24.4)$ & \\
\hline Phenotypic subtype & & & 0.334 \\
\hline Triple negative & $7(28)$ & $18(72)$ & \\
\hline HER2 over-expression & $5(20.8)$ & $19(79.2)$ & \\
\hline Luminal group & $16(16.2)$ & $83(83.8)$ & \\
\hline Unknown & $6(19.4)$ & $25(80.6)$ & \\
\hline
\end{tabular}

${ }^{\mathrm{a}}$ Values are expressed as mean \pm SD or No. (\%).

patients. The $\mathrm{pCR}$ rate was compared in these patients in terms of different phenotypic sub-types such as Triple Negative or TNBC (ER-/PR-/HER2-), HER2 over expression (ER/PR-/HER2+) and Luminal group (ER+/PR+/HER2-). There was no significant difference between $\mathrm{PCR}$ and non-pCR groups based on phenotypic subtypes $(\mathrm{P}=0.334)$. 


\section{Discussion}

The results of this study showed that pCR was the most observed in tumors with higher Ki67 and as such NAC may be more beneficial to these patients.

Several studies have shown that NAC is more effective in patients with pCR. In a histological evaluation, pCR was defined as absence of tumor residual in breast tissue and axilla. Clinical response evaluation is less judicable and depends on the examiner. But evaluation of pathologic response is measurable and could strongly indicate PCR or non-pCR. In this study, pCR was considered owing to its high accuracy. Several studies have reported pCR rate to be $3 \%-16 \%$ and clinical response to be $50 \%-70 \%(5,12,13)$. In the present study, pCR rate was $17 \%$ in terms of lack of invasive residual in the breast tissue and lymph nodes. Thus, this result is in line with those of similar studies (1). pCR was observed in $29.1 \%$ of breast tissue and $40.7 \%$ of axillary lymph nodes.

The mean and median ages were 45.4 and 45 years, respectively. There was no significant difference between the two groups in terms of age, indicating that age is not a determining factor for predicting pathological response. This result is in agreement with most studies $(2,14)$.

Seventy percent of patients were premenopausal at the time diagnosis which is acceptable according to the mean age. There was no significant difference between the two groups in terms of menopausal status.

In a study conducted by Migllieta et al. (14) in Italia, 55 patients with locally advanced breast cancer were evaluated. According to their results, the median age was 55 years and $38 \%$ of the patients were premenopausal.

In the present study, $29.9 \%$ of the patients were diagnosed with invasive ductal carcinoma, 5.3\% were lobular carcinoma and $1.8 \%$ were inflammatory carcinoma. Most studies have demonstrated similar results $(3,10)$.

The tumor grade distribution includes $64.9 \%$ for grade II and only 8.1\% for grade I. There was a significant difference between the two groups in terms of tumor grade. In a study by von Minckwitz et al. (15), the higher tumor grade was associated with higher pCR rate and this is not in agreement with the results of the present study. Also, the results of Migllieta et al. (14) are not in line with the findings of the present study.

Sixty-six percent of the patients were ER positive and $34 \%$ of the patients were ER negative. There was no significant difference between the two groups in terms of estrogen receptor. This difference was not significant even after the combination of estrogen and progesterone receptors as hormone receptors status. In this study, the positivity and the negativity of estrogen receptors were evaluated without considering the degree or intensity of posi- tivity. This could be one of the reasons for the different results obtained in the present study. Several studies have shown an inverse association between hormone receptors and pCR rate. In ECHO's trial, estrogen receptors status was the only effective factor on pCR. Thus this result is in agreement with those obtained by Berry et al. (6), Colleoni et al. (16) and Migllieta et al. (14). In numerous studies, receptors were not evaluated singly, but the phenotypic sub types of breast cancer were considered. Andrate et al. could not find an association between hormone receptors and $\mathrm{PCR}$ rate. But based on sub-groups categorization of patients, triple negative breast cancer patients had the most pCR rate. This finding is in contrast with the results of the present study. However, they considered residual carcinoma in situ as non-pathologic response.

Other effective predictor factor of pCR is HER2 receptor. HER2 positivity is considered when the result is +3 or when the FISH or CISH test result is in the indeterminate situation (HER2 $=2+$ ). In this study, there was no significant relationship between HER2 receptor and pCR. The results of HER2 were not available in 31 patients. Migllieta et al. (14) showed that the pCR rate was $54 \%$ in 55 locally advanced breast cancer patients. In their study, the sample size was lower than other studies and the patients achieved more pCR. They could not find any association between hormone receptors and tumor grade with PCR, but an association between HER2 receptors and Ki67 of more than $20 \%$ was found.

In the present study, the mean of NAC courses was 6 ( 3 - 8 courses). $81.2 \%$ of the patients received 6 or more than 6 NAC courses, which explain the relatively equal treatment in terms of NAC courses. No significant difference was found between the two groups in terms of NAC courses. In a study by Migllieta et al. (14) 54\% of patients received less than 4 NAC courses and there was no significant difference in terms of pCR and chemotherapy courses.

Seventy-four percent of patients received taxane-based regimen, which contains 4 courses of Adriamycin, $\mathrm{Cy}$ clophosphamide and then paclitaxel. In $27 \%$ of the patients chemotherapy regimen was non taxane-based and includes ACE (Adriamycin, Cyclophosphamide and Epirubucin), CF (Cyclophosphamide, Fluorouracil) and CMF (Cyclophosphamide, Methotrexate, Fluorouracil). In this study, taxane-based and non taxane-based chemotherapy regimen had no effect on pathologic response.

Several studies have shown that pCR rate have doubled by the addition of taxane to standard regimen containing anthracyclin. The different results obtained in the present study could be due to the less sample size.

Furthermore, in the present study, the mean of Ki67 level was 34.8 and the median was 25 (1-90). There was a significant difference between the Ki67 level and pCR rate. 
This indicates that tumors with Ki67 expression of more than $40 \%$ had significantly more pCR rate than tumors with Ki67 expression of less than $40 \%$. Ki67 is an antigen that is expressed in all phases of cell cycles except Go and shows the tumor cell proliferation rate. Numerous studies have shown the relationship between Ki67 rate and the outcome of breast cancer, as well as NAC response $(17,18)$.

Chemotherapy is more effective against tumors with higher proliferation rate which was confirmed in the present study. Therefore, ki67 expression can be introduced as an acceptable index for predicting the PCR rate after NAC.

There was no significant difference between the two groups after categorizing the patients based on phenotypic sub types, such as Triple Negative, HER2 overexpression and Luminal (A or B) groups; although pCR rate was $16.2 \%$ in Luminal group as compared with 28 and $20 \%$ pCR rates observed in TNBC and HER2+ patients, respectively. The Luminal A and B groups were merged due to absence of Ki67 data in the medical reports of patients.

Xiaoxian et al. (19) reported 104 pCR among 237 cases who received neoadjuvant chemotherapy (43.9\%). Among 229 cases with available biomarker information, 72 were luminal, 79 were HER2 and 78 were TNBC. They found that pCR was significantly higher in HER2 and triple-negative subtypes (58.2 and $47.4 \%$, respectively) as compared with luminal subtype (27.8\%). Also, they reported a strong correlation between pCR and Ki67 score both as a categorical variable (specifically when $<15 \%$ was set as the threshold for defining high proliferation index) and as a continuously increasing variable. These findings are similar to those of other studies.

However, their results of the correlation between PCR and breast cancer subtypes are different from findings of the present study. This difference may be related to the use of anti-HER2 therapies in the neoadjuvant setting. Also, the ratio of luminal, HER2+ and TNBC is different in their study, indicating that, in this study, the distribution of patients were nearly equal between the three groups, but the luminal subtype composed more than $50 \%$ of our patients.

From a biological standpoint, the relatively low pCR rates in the HR+/HER2- group strengthen the existing evidence that majority of these tumors are generally resistant to chemotherapy, and that efforts should be focused on alternative approaches of treatment and exploring ways to overcome this chemotherapy resistance. In addition, recent studies have demonstrated that patient outcomes are generally good for this subgroup of patients whether they achieve a pCR or not, indicating that the achievement of pCR may not be prognostic for survival for this particular breast cancer subtype. Von Minckwitz et al. (15) suggested that pCR may not be a suitable endpoint for the luminal subtypes. Specifically, they found that in low-proliferative subgroups (which included lobular histology, grade 1 and hormone receptor positive tumors), pCR conferred no predictive power in disease-free or overall survival. Thus this is in contrast with the high-proliferative subgroup (which included ductal histology, grade $2 / 3$, and hormone receptor negative tumors) in which pCR was associated with improved disease-free and overall survival.

Houssami et al. (11) in their meta-analysis of 30 studies that comprised 11,695 patients, estimated that pCR occurred in $8.3 \%$ of hormonal receptor positive HR+/HER2-, $18.7 \%$ of HR+/HER $2+, 38.9 \%$ of HR-/HER $2+$, and $31.1 \%$ of triple negatives. In 3 groups model (30 studies), the overall pooled estimate of $\mathrm{pCR} \%$ was $18.9 \%$ excluding subjects with unknown subtype, and tumor subtype was strongly associated with $\mathrm{pCR} \%(\mathrm{P}<0.0001)$.

The limitations of this study included the small sample size, the lack of patients' information and tumor characteristics such as hormone receptors, HER2 and Ki67. Studies with larger sample size and follow-up of patients to evaluate the overall and disease free survival is recommended.

The aim of this study was to assess the rate of pCR after NAC and to evaluate impacting factors in breast cancer patients who were referred to the breast cancer research center. Our findings showed that PCR was significantly associated with the level of Ki67 expression which is an indicator of tumor proliferation. Pathologic complete response can be considered a reasonable factor for predicting tumor response and prognosis of the patients. Longer follow-up of the patients to compare survival between PCR and nonpCR groups is recommended.

\section{Acknowledgments}

The authors thank the Motamed cancer institute for financial support and Dr Shahpar Haghighat for her statistical guidance.

\section{Footnotes}

Authors' Contribution: None declared. Conflict of Interest: None declared.

Financial Disclosure: None declared.

\section{References}

1. Mauri D, Pavlidis N, Ioannidis JP. Neoadjuvant versus adjuvant systemic treatment in breast cancer: a meta-analysis. J Natl Cancer Inst. 2005;97(3):188-94. doi: 10.1093/jnci/dji021. [PubMed: 15687361]. 
2. Guarneri V, Broglio K, Kau SW, Cristofanilli M, Buzdar AU, Valero V, et al. Prognostic value of pathologic complete response after primary chemotherapy in relation to hormone receptor status and other factors. J Clin Oncol. 2006;24(7):1037-44. doi: 10.1200/JCO.2005.02.6914. [PubMed: 16505422].

3. Mazouni C, Peintinger F, Wan-Kau S, Andre F, Gonzalez-Angulo AM, Symmans WF, et al. Residual ductal carcinoma in situ in patients with complete eradication of invasive breast cancer after neoadjuvant chemotherapy does not adversely affect patient outcome. J Clin Oncol. 2007;25(19):2650-5. doi: 10.1200/JCO.2006.08.2271. [PubMed: 17602071].

4. Chollet P, Amat S, Cure H. Prognostic significance of a complete pathological response after induction chemotherapy in operable breast cancer. Br J Cancer. 2002;86(7):1041-6.

5. Bolan PJ, Wey A, Eberly L. Assessing prognosis and therapy response in primary systemic therapy of breast cancer with magnetic resonance spectroscopy. Cancer Res. 2012;72(24):1-14.

6. Berry DA. Adaptive clinical trials in oncology. Nat Rev Clin Oncol. 2011;9(4):199-207. doi: 10.1038/nrclinonc.2011.165. [PubMed: 22064459].

7. Cortazar P, Zhang L, Untch M. Abstract S1-11: meta-analysis results from the collaborative trials in neoadjuvant breast cancer(CTNeoBC). Cancer Res. 2012;72(24):1-11. doi: 10.1038/sj/bjc/6600210.

8. Avril N, Sassen S, Roylance R. Response to therapy in breast cancer. J Nucl Med. 2009;50 Suppl 1:55S-63S. doi: 10.2967/jnumed.108.057240. [PubMed: 19380410].

9. Ring AE, Smith IE, Ashley S, Fulford LG, Lakhani SR. Oestrogen receptor status, pathological complete response and prognosis in patients receiving neoadjuvant chemotherapy for early breast cancer. BrJCancer. 2004;91(12):2012-7. doi: 10.1038/sj.bjc.6602235. [PubMed: 15558072]. [PubMed Central: PMC2409783].

10. Andrade DA, Zucca-Matthes G, Vieira RA, Andrade CT, Costa AM, Monteiro AJ, et al. Neoadjuvant chemotherapy and pathologic response: a retrospective cohort. Einstein (Sao Paulo).2013;11(4):446-50. [PubMed: 24488382]. [PubMed Central: PMC4880380].

11. Houssami N, Macaskill P, von Minckwitz G, Marinovich ML, Mamounas E. Meta-analysis of the association of breast cancer subtype and pathologic complete response to neoadjuvant chemotherapy. Eur J Cancer. 2012;48(18):3342-54. doi: 10.1016/j.ejca.2012.05.023.
[PubMed: 22766518].

12. Bear HD, Anderson S, Brown A, Smith R, Mamounas EP, Fisher B, et al. The effect on tumor response of adding sequential preoperative docetaxel to preoperative doxorubicin and cyclophosphamide: preliminary results from National Surgical Adjuvant Breast and Bowel Project Protocol B-27. J Clin Oncol. 2003;21(22):4165-74. doi: 10.1200/JCO.2003.12.005. [PubMed: 14559892].

13. Bardia A, Baselga J. Neoadjuvant therapy as a platform for drug development and approval in breast cancer. Clin Cancer Res. 2013;19(23):6360-70. doi: 10.1158/1078-0432.CCR-13-0916. [PubMed: 24298066].

14. Miglietta L, Venella P, Canobbio L, Parodi MA, Guglielmini P, Buccardo P. Clinical and pathological response to primary chemotherapy in patients with locally advanced breast cancer grouped according to hormonal receptors, Her2 status, grading and Ki-67 proliferation index. Anticancer Res. 2009;29(5):1621-5.

15. von Minckwitz G, Untch M, Blohmer JU, Costa SD, Eidtmann $H$, Fasching PA, et al. Definition and impact of pathologic complete response on prognosis after neoadjuvant chemotherapy in various intrinsic breast cancer subtypes. J Clin Oncol. 2012;30(15):1796-804. doi 10.1200/JCO.2011.38.8595. [PubMed: 22508812].

16. Colleoni M, Viale G, Goldhirsch A. Lessons on responsiveness to adjuvant systemic therapies learned from the neoadjuvant setting. Breast. 2009;18 Suppl 3:S137-40. doi: 10.1016/S0960-9776(09)70289-9. [PubMed: 19914533].

17. Petit T, Wilt M, Velten M, Millon R, Rodier JF, Borel C, et al. Comparative value of tumour grade, hormonal receptors, Ki-67, HER-2 and topoisomerase II alpha status as predictive markers in breast cancer patients treated with neoadjuvant anthracycline-based chemotherapy. Eur J Cancer. 2004;40(2):205-11. [PubMed:14728934].

18. Fasching PA, Heusinger K, Haeberle L, Niklos M, Hein A, Bayer CM et al. Ki67, chemotherapy response, and prognosis in breast cancer patients receiving neoadjuvant treatment. BMC Cancer. 2011;11:486. doi: 10.1186/1471-2407-11-486. [PubMed: 22081974]. [PubMed Central: PMC3262864].

19. Li XB, Krishnamurti U, Bhattarai S, Klimov S, Reid MD, O'Regan R, et al. Biomarkers Predicting Pathologic Complete Response to Neoadjuvant Chemotherapy in Breast Cancer. Am JClin Pathol. 2016;145(6):8718. doi: 10.1093/ajcp/aqw045. [PubMed: 27298399]. 